\title{
Targeting A549 lung adenocarcinoma cell growth and invasion with protease-activated receptor-1 siRNA
}

\author{
ZHUO WU ${ }^{1}$, YANYAN ZENG ${ }^{2}$, MINGKANG ZHONG $^{1}$ and BIN WANG ${ }^{1}$ \\ ${ }^{1}$ Department of Pharmacy, Huashan Hospital, Fudan University, Shanghai 200040; ${ }^{2}$ Department of Pharmacy, \\ Teaching Hospital of Fujian Medical University, Quanzhou Women's and Children's Hospital, \\ Quanzhou, Fujian 362000, P.R. China
}

Received August 13, 2013; Accepted February 11, 2014

DOI: $10.3892 / \mathrm{mmr} .2014 .2023$

\begin{abstract}
Lung cancer is the major cause of cancer-associated mortality worldwide and the invasive and metastatic characteristics of lung tumor cells are responsible for their high malignancy. Protease-activated receptor 1 (PAR1) is a G-protein-coupled receptor (GPCR) which is activated by a unique proteolytic mechanism. PARs have crucial roles in hemostasis and thrombosis as well as tumor progression. RNA interference (RNAi) is a fundamental cellular mechanism for gene silencing that is able to be harnessed for the development of novel anti-cancer drugs. In the present study, PAR1 was successfully inhibited by using Lipofectamine RNAiMAX transfection reagent to deliver siRNA. Inhibition occurred at the mRNA and protein level as determined by polymerase chain reaction and western blot analysis. Furthermore, the growth and invasion of tumor cells were significantly decreased. In conclusion, the present study demonstrated that the progression of A549 cells is able to be inhibited by knockdown of PAR1 expression. Efficient delivery of the specific siRNA targeting PAR1 may be used for further study in clinical cancer therapy.
\end{abstract}

\section{Introduction}

Lung cancer is the major cause of cancer-associated mortality worldwide, with non-small cell lung cancer (NSCLC) histology predominating over small cell lung cancer (SCLC). The invasive and metastatic characteristics of lung tumor cells are responsible for their high malignancy. Patients with lung cancer frequently exhibit tumor cell invasion and metastasis prior to diagnosis, which renders current treatments, including surgery, radiotherapy and chemotherapy ineffective. Typically,

Correspondence to: Professor Bin Wang, Department of Pharmacy, Huashan Hospital, Fudan University, 12 Middle Wulumuqi Road, Shanghai 200040, P.R. China

E-mail: shhswangbin@126.com

Key words: RNAi, protease-activated receptor 1, siRNA, lung cancer the 5-year survival rate following diagnosis is $<20 \%$. Therefore, it is important to study the molecular basis of lung cancer cell invasion and metastasis in order to design novel therapeutic agents that are able to decrease the malignancy of lung cancer (1).

Protease-activated receptors (PARs) are G-protein-coupled receptors (GPCRs) that signal in response to extracellular protease. There are four human PARs (PAR1-4) which have impotant roles in hemostasis and thrombosis as well as in inflammatory and proliferative response (2). PAR1 was originally dubbed the thrombin receptor since it was first found in a search for a receptor that confers thrombin signaling on human platelets and other cell types $(3,4)$. Unlike typical ligand-receptor interactions, thrombin cleaves the $\mathrm{NH}_{2}$ terminus of PAR1 at serine $42\left(\mathrm{Ser}^{42}\right)$. Upon cleavage, the new $\mathrm{NH}_{2}$-terminal peptide acts as a tethered ligand that activates the receptor and initiates cellular signaling (5).

PAR1 is overexpressed in aggressive melanoma as well as colon, prostate and invasive breast cancers (6-9). Previous studies showed that the upregulation of PAR1 is strongly associated with low survival rates in patients with gliomas (10), breast cancer (11) and primary gallbladder carcinoma (12).

The present study focused on the downregulation of PAR1 expression by small interfering ribonucleic acids (siRNAs). By using siRNA and Lipofectamine RNA interference (RNAi) MAX complex formation in vitro, silencing was achieved at the protein level as demonstrated by western blot analysis and at the mRNA level as shown by polymerase chain reaction (PCR). Furthermore, the growth and metastasis of A549 cells were decreased. PAR1 may be a promising drug target in clinical cancer therapy.

\section{Materials and methods}

Cell lines and culture. The A549 cell line was obtained from Keygen Biotech (Nanjing, China) and cultured in RPMI-1640 medium (Invitrogen, Carlsbad, CA, USA) containing 10\% fetal bovine serum (FBS; TianHang Biological Technology Co., Ltd., Hangzhou, China).

siRNAs. The sequences of three siRNA duplexes were purchased from GenePharma, Shanghai, China. siRNA1 (5'-GAC ACU CUU UGU CCC AUC UTT-3'), siRNA2 
(5'-CUG UCA UGA UGU GCU CAA UTT-3') and siRNA3 (5'-GGC AGU UGA UGG CAA GUA ATT-3') were designed to target different coding regions of the human PAR1 mRNA sequence (GeneBank accession no. NM_2149). A BLAST (NCBI database; National Center for Biotechnology Information, Bethesda, MD, USA) search was performed to confirm the only targets of the three duplexes on PAR1. A negative control (5'-UUC UCC GAA CGU GUC ACG UTT-3') and a positive control (GAPDH, 5'-GUA UGA CAA CAG CCU CAA GTT-3') were also obtained from GenePharma.

Efficiency of delivery in vitro. A549 cells were seeded in 6-well plates with RPMI-1640 containing 10\% FBS without antibiotics and allowed to attach overnight. The following day, the cells were transfected with the fluorescein amidite (FAM; GenePharma, Shanghai, China)-labeled negative control siRNA, according to the manufacturer's instructions for Lipofectamine RNAiMAX transfection reagent (Invitrogen Life Technologies, Carlsbad, CA, USA) -based transfections when the confluence was $60-80 \%$. Six hours following transfection, the 6-well plates were observed under a fluorescence microscope (Axiovert $40 \mathrm{CFL}$; Carl Zeiss, Jena, Germany) to observe the fluorescence (green, negative control FAM). The final concentration of the Lipofectamine RNAiMAX transfection reagent was $0.2 \%(5 \mu \mathrm{l})$. The final concentration of the negative control FAM was $100 \mathrm{nM}$.

Transfection with siRNAs in vitro. Cells were transfected with siRNAs by the aforementioned method. The final concentration of siRNAs was $100 \mathrm{nM}$. A control, a negative control and a GAPDH-positive control group were also contained in the 6-well plates.

$P C R$. Total RNA was extracted from the cells with TRIzol reagent (Invitrogen) according to the manufacturer's instructions and quantified by ultraviolet absorbance spectroscopy. Reverse transcription was performed using $500 \mathrm{ng}$ of total RNA. The reaction mixture contained 5X PrimeScript ${ }^{\circledR}$ buffer (Takara, Dalian, China), total RNA and RNase-free water, and the reaction was performed according to the manufacturer's instructions of the PrimeScript ${ }^{\circledR}$ RT Master Mix Perfect Real Time (Takara). Relative quantitative analysis of the cDNA was performed using the ABI PRISM ${ }^{\circledR} 7500$ (Applied Biosystems, Grand Island, NY, USA) and the SYBR $^{\circledR}$-Green Premix Ex Taq ${ }^{\mathrm{TM}}$ kit (Takara) according to the manufacturer's instructions. PCRs were performed in a total volume of $20 \mu \mathrm{l}$, including $2 \mu \mathrm{l} \mathrm{cDNA}$ and $0.2 \mu \mathrm{M}$ primers. The primers used were: PAR1, sense, 5'-GTG ATT GGC AGT TTG GGT CT-3' and antisense, 5'-GCC AGA CAA GTG AAG GAA GC-3'; GAPDH, sense, 5'-CAG TCC ATG CCA TCA CTG CCA-3' and antisense, 5'-CAG TGT AGC CCA GGA TGC CCT T-3'. Amplification was conducted at $95^{\circ} \mathrm{C}$ for $30 \mathrm{sec}$, then 40 cycles at $95^{\circ} \mathrm{C}$ for $5 \mathrm{sec}$ and $60^{\circ} \mathrm{C}$ for $30 \mathrm{sec}$. At the end of each PCR, melting curve analysis was performed to confirm that the amplified product was specific. All the reactions were performed in triplicate. Sample values were normalized to the expression of the housekeeping gene GAPDH, and the relative expression was calculated using the AB 7500 system SDS software (Applied Biosystems).
Western blot analysis. Cell extracts were prepared with $200 \mu 1$ mixture of radioimmunoprecipitation assay (RIPA) lysis buffer (Beyotime, Jiangsu, China) and $1 \mathrm{mM}$ phenylmethylsulfonyl fluoride. The total protein was extracted. Samples containing equivalent amounts of protein $(20 \mu \mathrm{g})$ were applied to a $10 \%$ SDS-PAGE gel by electrophoresis. The separated proteins were transferred onto polyvinylidene fluoride membranes (Millipore, Bedford, MA, USA) and incubated overnight at $4^{\circ} \mathrm{C}$. Blotting membranes were blocked for $1 \mathrm{~h}$ at room tempreture, washed three times, and then incubated with mouse anti-PAR1 (1:100; Santa Cruz Biotechnology, Inc., Santa Cruz, CA, USA) in Tris-buffered saline and Tween 20 (TBST) overnight at $4^{\circ} \mathrm{C}$. GAPDH antibodies (1:2,000; KangChen Biotech, Shanghai, China) were used as an internal control. Following several washes with TBST buffer, the membranes were incubated for $2 \mathrm{~h}$ with horseradish peroxidase-linked secondary antibody (1:1,000; Jackson ImmunoResearch Laboratories, Inc., West Grove, MA, USA). The membranes were then processed with enhanced chemiluminescence western blotting detection reagents (Pierce, Rockford, IL, USA). Chemifluorescence was detected using the ChemiDoc ${ }^{\mathrm{TM}}$ XRS+ imaging system (Bio-Rad, Hercules, CA, USA).

Cell viability assays. Cell viability was measured by the WST-8 assay following optimized manufacturer's instructions (Dojindo, Kumamoto, Japan). Briefly, one day prior to transfection, the A549 cells were seeded at a density of $5,000 \mathrm{cells} / 100 \mu \mathrm{l} /$ well in 96 -well culture plates and incubated in a humidified incubator at $37^{\circ} \mathrm{C}$. The cells were then treated with PAR1 siRNA at five different concentrations $(0,10,25$, 50 and $100 \mathrm{nM})$. A negative control group was also included. Following $48 \mathrm{~h}$ of incubation, $10 \mu \mathrm{l}$ 2-(2-methoxy-4-nitroph enyl)-3-(4-nitrophenyl)-5-(2,4-disulfophenyl)-2H-tetrazolium (WST-8) was added to each well. The cells were then incubated for $2 \mathrm{~h}$ prior to measuring the optical density (OD) at $540 \mathrm{~nm}$. Each group contained five duplicates. The percentage of viable cells was determined using the formula: Ratio $(\%)=[\mathrm{OD}$ (treatment) - OD (blank)/OD (control) - OD (blank)] x 100.

Wound healing. A549 cells were seeded at $3 \times 10^{6}$ cells/well in 6-well plates. A linear wound was generated in the monolayer with a sterile $10 \mu 1$ plastic pipette tip. The experiment was performed on PAR1 siRNA-transfected, negative siRNA-transfected and control groups. After 0,12, 24, 48 and $72 \mathrm{~h}$ of incubation, images of the cells were captured by the TE2000 Nikon microscope (Nikon Corporation, Tokyo, Japan), using NIS-Elements F software, version 3.0 (Nikon Corporation). The mobility was calculated using the formula: Mobility $=\left(\right.$ Width $_{0 \mathrm{~h}}$ group - Width $\left._{\mathrm{x} \mathrm{h} \mathrm{group}}\right) /$ Width $_{0 \mathrm{~h} \mathrm{group}} \mathrm{x} 100 \%$.

Cell migration. Transwell chambers (Costar, Bethesda, MD, USA) were used for the cell mobility experiments. The experimental group which had been transfected with PAR1 siRNA for $24 \mathrm{~h}$, as well as the positive and negative control groups, were incubated into the upper compartment of the Transwell chambers, respectively, at a density of $1 \times 10^{5} / \mathrm{ml}$ and $100 \mu \mathrm{l} /$ well. The cells were incubated at $37^{\circ} \mathrm{C}$ for $12 \mathrm{~h}$. Cells that did not penetrate the membrane were wiped off. The membrane was removed, fixed with paraformaldehyde 

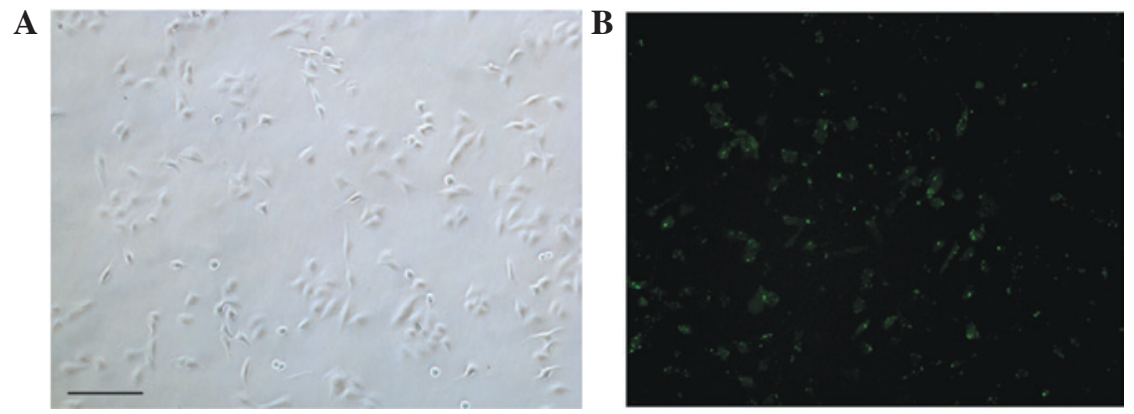

Figure 1. Delivery efficiency of negative control-FAM in A549 cells. Fluorescence microscopy images of A549 cells treated with negative control-FAM using the Lipofectamine RNAiMAX transfection reagent. Six hours following transfection, the plates were observed under (A) visible light and (B) a fluorescence microscope (scale bar, $100 \mu \mathrm{m})$. FAM, fluorescein amidite.

A

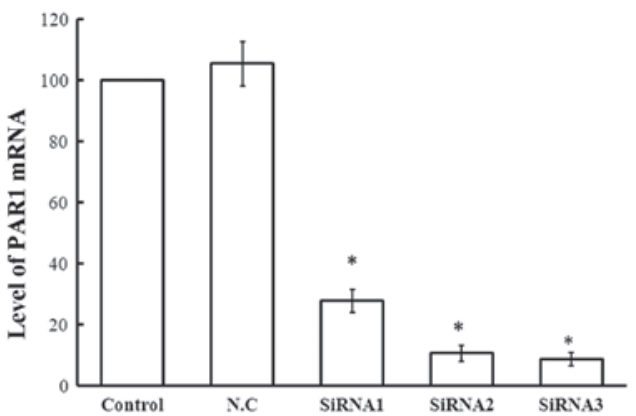

$\mathbf{B}$
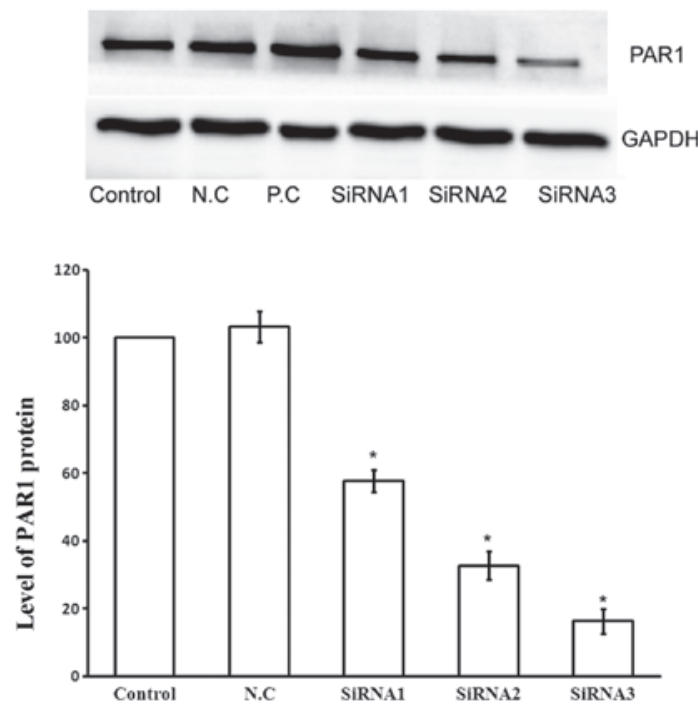

Figure 2. Levels of PAR1 expression following transfection with siRNAs $(100 \mathrm{nmol} / \mathrm{l})$. (A) Column diagram shows the levels of PAR1 mRNA in A549 cells 24 h following transfection with the three different siRNA dulplexes examined by PCR. Bars 1, Control; 2, N.C.; 3, siRNA1; 4, siRNA2; 5, siRNA3. (B) Effects of the three siRNA duplexes on PAR1 protein expression examined by western blot analysis $48 \mathrm{~h}$ following transfection. The column diagram shows PAR1 protein levels in A549 cells following transfection. ${ }^{*} \mathrm{P}<0.05$ compared with untreated control cells. Experiments were performed at least three times and a representative experiment is shown in B. The values shown in A and B are the mean \pm standard deviation of three independent experiments. PAR1, protease-activated receptor 1; mRNA, messenger ribonucleic acid; siRNA, small interfering ribonucleic acid; PCR, polymerase chain reaction; N.C., negative control.

and stained with $0.1 \%$ crystal violet. Five fields of view were randomly selected and the number of cells that penetrated the membrane was counted. The mobility inhibition rate was calculated using the equation: Mobility inhibition rate $=$ (the number of cells in the control group that penetrated the membrane - the number of cells in the PAR1 siRNA group that penetrated the membrane)/the number of cells in the control group that penetrated the membrane $\mathrm{x} 100 \%$.

Cell invasion. Transwell chambers were used to determine the cell invasiveness. The membrane at the bottom of the Transwell chamber was evenly coated with $50 \mu$ l diluted Matrigel. Cells from the experimental group which had been transfected with PAR1 siRNA for $24 \mathrm{~h}$ as well as the positive and negative control groups were inoculated into the upper compartment of the Transwell chambers at a density of $1 \times 10^{5}$ cells $/ \mathrm{ml}$ and $100 \mu \mathrm{l} /$ well. The cells were incubated at $37^{\circ} \mathrm{C}$ for $24 \mathrm{~h}$. Cells that did not penetrate the polycarbonate membrane were wiped off. The membrane was then fixed with paraformaldehyde and stained with $0.1 \%$ crystal violet. Five fields of view were randomly selected and the number of cells that penetrated the membrane was counted. The invasion inhibition rate was calculated using the formula: Invasion inhibition rate $=$ (the number of cells in the control group that penetrated the membrane - the number of cells in the experimental group that penetrated the membrane)/the number of cells in the control group that penetrated the membrane x $100 \%$.

Statistical analyses. The PCR and western blot data were normalized to the GAPDH controls. The results were expressed as the mean \pm standard deviation and the significance of differences was determined using one-way analysis of variance (ANOVA) followed by Scheffe's post hoc test. Differences with $\mathrm{P}<0.05$ were considered to be statistically significant.

\section{Results}

Efficiency of delivery. The A549 cells were seeded in 6-well plates and incubated overnight. The following day, the cells were transfected with $12.5 \mu \mathrm{l}$ negative control-FAM. Six hours following transfection, the 6-well plates were observed under a fluorescence microscope to observe green fluorescence resulting from the negative control-FAM. As shown in Fig. 1, the delivery efficiency to A549 cells, which was $95 \%$, was sufficiently high to transfect siRNAs into A549 cells in the present study. 


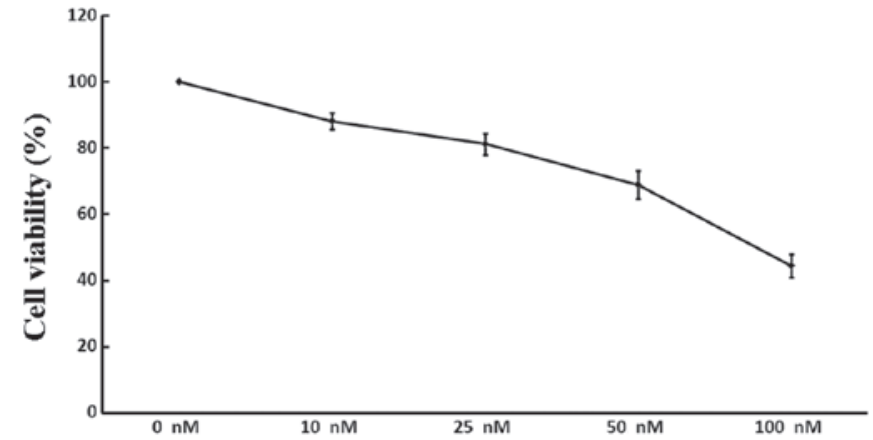

Figure 3. WST-8 assay on A549 cell viability. The cells were treated with 0 , $10,25,50$ and $100 \mathrm{nM}$ siRNA. Following $48 \mathrm{~h}$ of incubation post-treatment, $10 \mu \mathrm{lWST}-8$ was added to each well and incubated for $2 \mathrm{~h}$ prior to measuring the $\mathrm{OD}$ at $540 \mathrm{~nm}$. The values shown are the mean \pm standard deviation of three independent experiments. WST-8, 2-(2-methoxy-4-nitrophenyl)-3-(4-n itrophenyl)-5-(2,4-disulfophenyl)-2H-tetrazolium; siRNA, small interfering ribonucleic acid; OD, optical density.

Inhibitory effect of three siRNA duplexes on PARI expression. To examine the silencing effect of siRNAs on PAR1 mRNA and protein, three siRNA duplexes, a positive control and a negative control at the same final concentration of $100 \mathrm{nM}$ were used for transfection of A549 cells with $5 \mu \mathrm{l}$ Lipofectamine RNAiMAX transfection reagent. Following 24 $\mathrm{h}$ of incubation, the cells were collected for PCR. As shown in Fig. 2A, compared with the control, all three duplexes significantly decreased PAR1 mRNA levels $(\mathrm{P}<0.05)$. However, siRNA2 and siRNA3, which led to $\sim 89.3$ and $91.3 \%$ decrease of PAR1 mRNA, respectively, exerted a greater silencing effect compared with siRNA1 $(72.3 \%, \mathrm{P}<0.05)$. Following $48 \mathrm{~h}$ of incubation post-transfection, the cells were collected for western blot analysis. As shown in Fig. 2B, siRNA3, which caused an $\sim 83.6 \%$ decrease of PAR1 protein, had the most marked silencing effect compared with siRNA1 and siRNA2. Transfection of the negative control did not decrease the mRNA or the protein levels of the PAR1 gene and transfection of the positive control decreased both the mRNA and protein levels of the GAPDH gene. From the above results, siRNA3 was proven to have the most marked silencing effect among the three siRNAs assessed. Accordingly, siRNA3 was selected to be used in the present study.

PAR1 siRNA3 suppresses A549 cell viability. The viability of A549 cells following treatment with increasing concentrations of siRNA3 $(0,10,25,50$ and $100 \mathrm{nM})$ was assessed. As demonstrated by the WST-8 assay (Fig. 3), siRNA3 decreased the quantity of viable cells in a dose-dependent manner: Following incubation with $100 \mathrm{nM}$ siRNA, the number of A549 cells was reduced by $55.5 \%$, whereas siRNA at a lower concentration $(10 \mathrm{nM})$ exerted only a minor inhibitory effect $(11.9 \%)$. There was no significant difference between the negative and positive controls $(\mathrm{P}>0.05)$.

In order to investigate the role of PAR1 in the viability of A549 cells, PAR1 mRNA levels were assessed $48 \mathrm{~h}$ following transfection with siRNA3 at various concentrations. As shown in Fig. 4, $10 \mathrm{nM}$ siRNA3 decreased PAR1 mRNA levels by $32.5 \%$, while $100 \mathrm{nM}$ siRNA3 led to a $76.1 \%$ decrease in PAR1 mRNA levels. Thus, siRNA3 decreased PAR1 mRNA levels in a dose-dependent manner, affecting the viability of A549 cells.

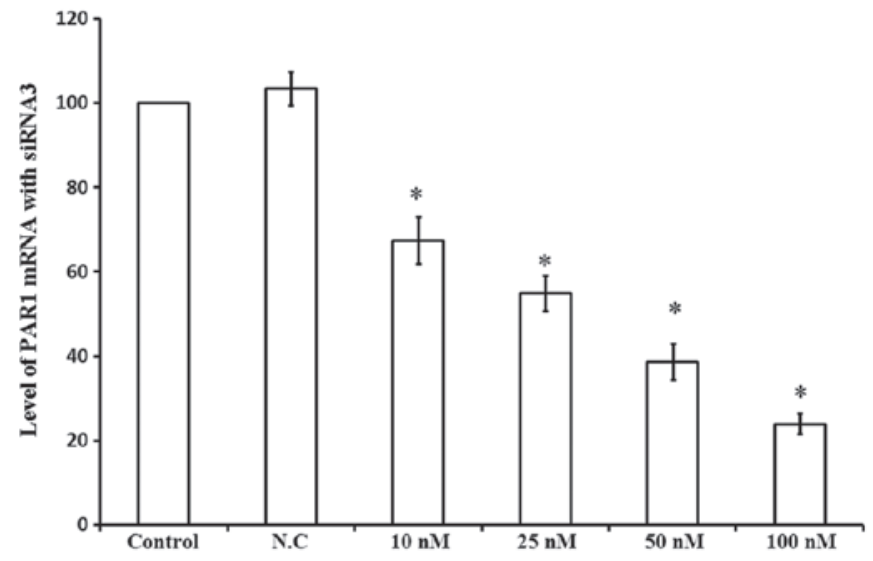

Figure 4. Levels of PAR1 mRNA following transfection with different concentrations of siRNA3. Column diagram shows the levels of PAR1 mRNA in A549 cells $48 \mathrm{~h}$ following transfection with four different concentrations of siRNA3 examined by Bars PCR. 1, Control; 2, N.C.; 3, 10 nM; 4, 25 nM; 5, 50 $\mathrm{nM} ; 6,100 \mathrm{nM} .{ }^{*} \mathrm{P}<0.05$ compared with control cells. PAR1, protease-activated receptor 1; mRNA, messenger ribonucleic acid; siRNA, small interfering ribonucleic acid PCR, polymerase chain reaction; N.C., negative control.

PAR1 siRNA inhibits the migration of A549 cells. As demonstrated in the wound healing experiment (Fig. 5A), PAR1 siRNA inhibited the migration of A549 cells within $72 \mathrm{~h}$ post-perforation of the cell layer at $100 \mathrm{nM}$. In the Transwell chamber experiment (Fig. 5B), a significant decrease in migration $(63.6 \%)$ was observed between the cells penetrated from the control and the treated groups.

PAR1 siRNA inhibits the invasiveness of A549 cells. As shown in Fig. 6, PAR1 siRNA visibly inhibited the invasiveness of the cells by $67.6 \%$, at $100 \mathrm{nM}$ compared with the control group. These results suggest that PAR1 has a role in promoting the invasive phenotype of A549 adenocarcinoma cells. A significant difference $(\mathrm{P}<0.05)$ was observed between the control and treatment groups.

\section{Discussion}

PAR1, the prototype of the PAR family, was originally recognized to transmit cellular responses to thrombin, the main effector protease of the coagulation cascade (3). Subsequently, PAR1 was identified to be involved in tumor progression. Bar-Shavit et al (13) reported that in epithelial malignancies, by recruiting the dishevelled homolog (DVL), an upstream signaling partner of the canonical wingless type mouse mammary tumor virus integration site (Wnt) signaling pathway, PAR1, is able to eventually cause $\beta$-catenin stabilization, a core event in both tumorigenesis and developmental processes. Tantivejkul et al (14) proved that PAR1 is able to activate the necrosis factor $\mathrm{kB}(\mathrm{NF}-\mathrm{kB})$ signaling pathway, which finally results in the growth of prostate cancer cells. Additionally, PAR1 is a regulator of several genes and molecules involved in tumor growth and metastatic progression, including the vascular endothelial growth factor (VEGF), interleukin 8 (IL-8), and matrix metalloproteinases (MMPs) $(15,16)$.

Although the aforementioned studies support the fact that PAR1 is an important tumor-associated gene, the precise 
A
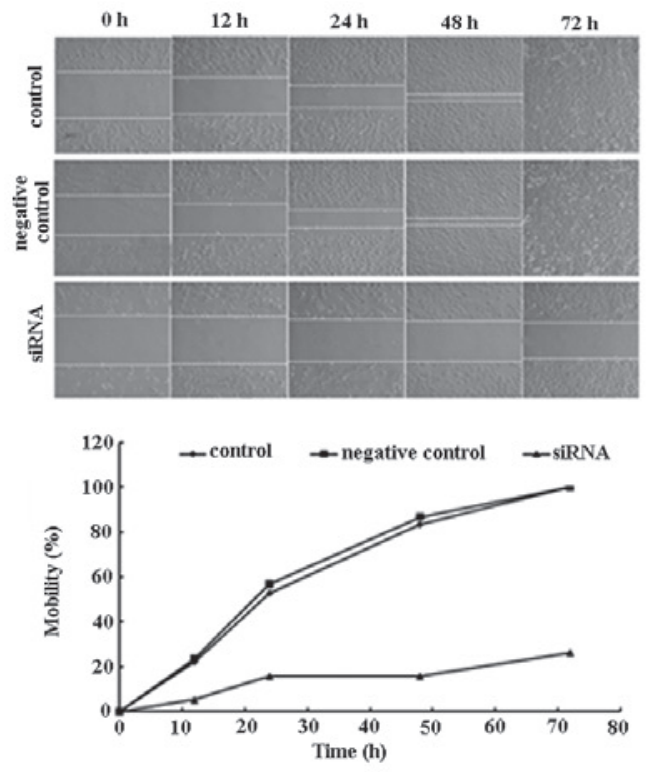

B
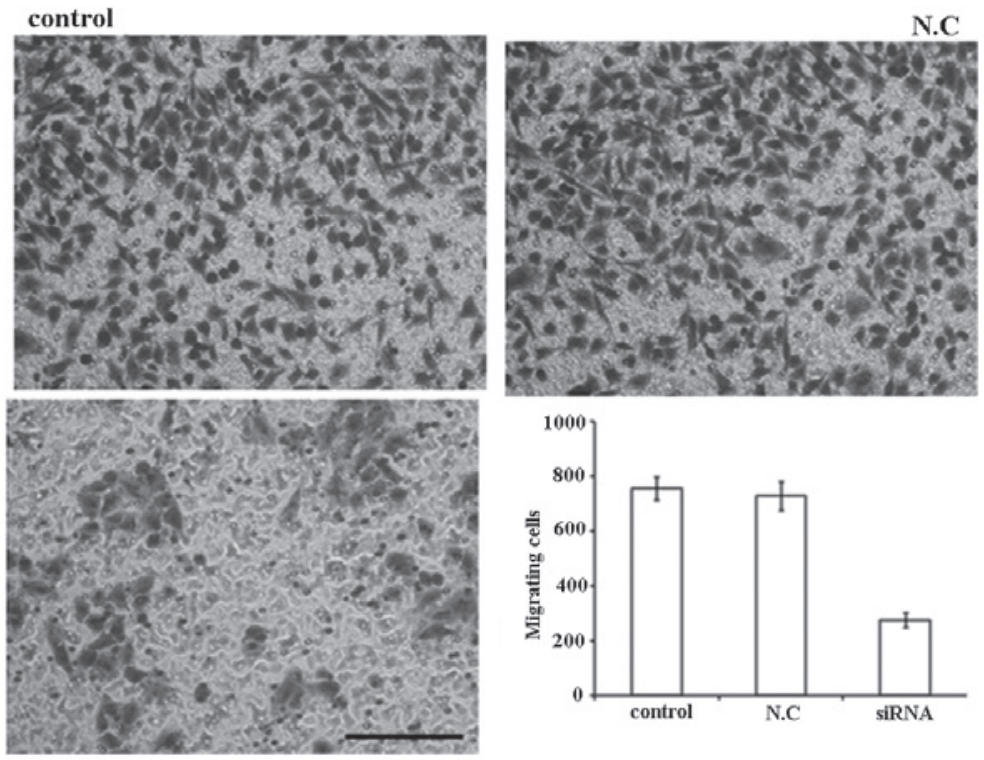

Figure 5. siRNA (100 nM) inhibits the mobility of A549 cells. (A) Migration ability of the control group, negative control group and siRNA transfection group $0,12,24,48$ and $72 \mathrm{~h}$ following perforation of the A549 cell monolayer with a sterile $10 \mu 1$ plastic pipette tip. The graph shows the mobility of cells in three groups at different time-points following perforation. (B) Cell migration evaluated using Transwell chambers. Cells that migrated through the pores to the lower surface of the membrane were fixed, stained and counted. Representative images of the membrane surface in the three groups (control, N.C., siRNA-transfected). Column diagram shows the quantification of the cell migration results. Each bar represents the mean \pm standard deviation of the counts from three independent experiments. Values for siRNA-treated cells versus control show significant differences. Scale bars, $100 \mu$ m. siRNA, small interfering ribonucleic acid; N.C, negative control.

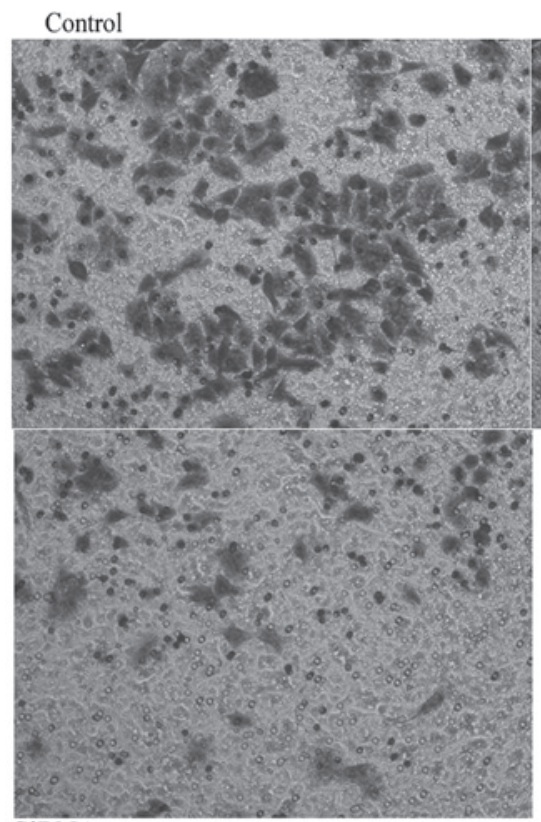

N.C.

SiRNA

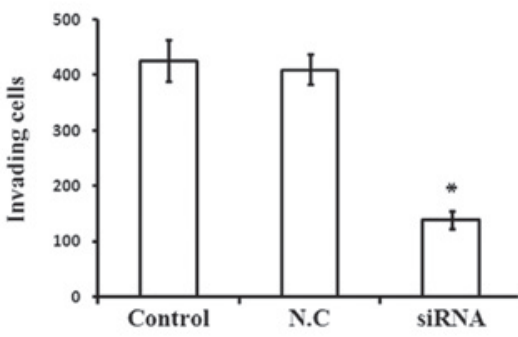

Figure 6. Effect of $100 \mathrm{nM}$ siRNA on the invasiveness of A549 cells. PAR1-depletion inhibits cell invasion as assayed in 24-well Matrigel invasion chambers, with incubation for $24 \mathrm{~h}$ with $10 \%$ serum as the chemoattractant. Cells that invaded the Matrigel-coated filter were fixed and stained with $0.1 \% \mathrm{crystal}$ violet. Representative images of the surfaces of the membranes of the three groups (control, N.C., siRNA-transfected). Column diagram shows the quantification of the cell invasion results. The values shown are the mean \pm standard deviation of three independent experiments. Values for siRNA-treated cells versus control are significantly different. Scale bars, $100 \mu \mathrm{m}$. PAR1, protease-activated receptor 1; siRNA, small interfering ribonucleic acid; N.C, negative control.

mechanism of its contribution to tumor progression remains to be elucidated. Recently, MMP-1 was reported to proteolytically activate PAR1 (17). In addition, MMP-1 has been identified as one of the most upregulated proteins in various types of cancer, including breast, esophageal and colorectal carcinomas (18-20). Silencing of MMP1-PAR1 signaling may 
improve the outcome of Taxotere treatment in advanced, metastatic breast cancer (21).

All of these findings suggest that the inhibition of PAR1 is beneficial to patients with tumors. RNAi is a tool which is able to silence genes in a sequence-specific manner. Following the finding that RNAi is mediated by long, double-stranded RNA in Caenorhabditis elegans in 1998 and the revelation of synthetic siRNAs being able to silence target genes in mammalian systems in 2001, there has been a large number of reports on therapeutic applications harnessing RNAi. Numerous cancer targets for RNAi therapies have been found in previous studies and by using RNAi, cancer therapy or its outcome may be improved (22-26).

In the present study, siRNA3 decreased PAR1 mRNA levels by $91.3 \%$ as determined by PCR and PAR1 protein levels were decreased by $83.6 \%$ as determined by western blot analysis. Furthermore, the present study provided substantial evidence for the role of PAR1 in survival, invasiveness and the metastatic capabilities of the A549 lung adenocarcinoma cell line. By silencing PAR1 with RNAi, the migration ability of A549 cells was inhibited by $63.6 \%$, invasion was decreased by $67.6 \%$ and viability was only $44.5 \%$ of the control group.

The diffusion of the tumor cells from the primary site and the infiltration of the extracellular matrix (ECM) were two significant steps in tumor invasion and metastasis, which are hallmarks of malignant tumors and are the major causes of mortality of patients with cancer. Besides PAR1, MMP and urokinase-type plasminogen activator (UPA) also participate in basement membrane destruction. RNA-interfering technology which targets these proteins in these pathways may contribute to favourable cancer prognosis.

The principal advantage of RNAi is that all targets, are theoretically druggable with RNAi, since any transcript that encodes a protein that causes or contributes to a disease is able to be targeted by RNAi $(27,28)$. This includes 'undruggable' targets which are, due to their structure and location, not accessible by other therapeutics.

Efficient delivery to targeted tissues is the main issue in developing RNAi as therapeutics. Both the non-viral delivery of siRNAs and viral delivery of shRNAs are being advanced as potential RNAi-based therapeutic approaches. Viral delivery approaches include retroviral, lentiviral, adenoviral and adeno-associated viral vectors. With regard to non-viral delivery, liposomes, lipid complexes or conjugates with small molecules (polymers, proteins and antibodies), electroporation and hydrodynamic gene transfer have all been used to facilitate the delivery of siRNAs to target cells.

Electroporation (EP) has been extensively used for drugs and plasmid delivery in a large number of organs and tissues (25,29-31). By selecting appropriate electrical parameters and electrodes, gene transfer may be optimized and tissue injury minimized. However, electroporation is often limited to tumors that are accessible and it is not possible to use it for the treatment of deep tumors, currently, only electrodes for the treatment of cutaneous and subcutaneous tumours have been designed and produced, including needle electrodes and plate electrodes. It is aspired that in the future, the development of technologies including microelectrodes may be beneficial for cancer therapy.

In conclusion, in the present study PAR1 was proven to be a significant target for clinical cancer therapy and additionally provides a novel target in small-molecular drug design. With the rapid progression of research and development of applications, RNAi may remain a significant class of therapeutics in the foreseeable future.

\section{References}

1. Wang Y, Yang H, Liu H, Huang J and Song X: Effect of staurosporine on the mobility and invasiveness of lung adenocarcinoma A549 cells: an in vitro study. BMC Cancer 9: 174, 2009.

2. Arora P, Ricks TK and Trejo J: Protease-activated receptor signalling, endocytic sorting and dysregulation in cancer. J Cell Sci 120: 921-928, 2007.

3. Rasmussen UB, Vouret-Craviari V, Jallat S, Schlesinger Y, Pagès G, Pavirani A, Lecocq JP, Pouysségur $J$ and Van Obberghen-Schilling E: cDNA cloning and expression of a hamster alpha-thrombin receptor coupled to $\mathrm{Ca}^{2+}$ mobilization. FEBS Lett 288: 123-128, 1991.

4. Vu TK, Hung DT, Wheaton VI and Coughlin SR: Molecular cloning of a functional thrombin receptor reveals a novel proteolytic mechanism of receptor activation. Cell 64: 1057-1068, 1991.

5. Villares GJ,Zigler M, Wang H, et al: Targeting melanoma growth and metastasis with systemic delivery of liposome-incorporated protease-activated receptor-1 small interfering RNA. Cancer Res 68: 9078-9086, 2008.

6. Tellez C and Bar-Eli M: Role and regulation of the thrombin receptor (PAR-1) in human melanoma. Oncogene 22: 3130-3137, 2003.

7. Chay CH, Cooper CR, Gendernalik JD, Dhanasekaran SM, Chinnaiyan AM, Rubin MA, Schmaier AH and Pienta KJ: A functional thrombin receptor (PAR1) is expressed on bone-derived prostate cancer cell lines. Urology 60: 760-765, 2002.

8. Even-Ram S, Uziely B, Cohen P, Grisaru-Granovsky S, Maoz M, Ginzburg Y, Reich R, Vlodavsky I and Bar-Shavit R: Thrombin receptor overexpression in malignant and physiological invasion processes. Nat Med 4: 909-914, 1998.

9. Darmoul D, Gratio V, Devaud H,Lehy T and Laburthe M: Aberrant expression and activation of the thrombin receptor protease-activated receptor-1 induces cell proliferation and motility in human colon cancer cells. Am J Pathol 162: 1503-1513, 2003

10. Zhang Y, Zhan H, Xu W, Yuan Z, Lu P, Zhan L and Li Q: Upregulation of matrix metalloproteinase-1 and proteinase-activated receptor-1 promotes the progression of human gliomas. Pathol Res Pract 207: 24-29, 2011.

11. Hernández NA, Correa E, Avila EP, Vela TA and Pérez VM: PAR1 is selectively over expressed in high grade breast cancer patients: a cohort study. J Transl Med 7: 47, 2009.

12. Du X, Wang S, Lu J, et al: Correlation between MMP1-PAR1 axis and clinical outcome of primary gallbladder carcinoma. Jpn J Clin Oncol 41: 1086-1093, 2011.

13. Bar-Shavit R, Turm H, Salah Z, Maoz M, Cohen I, Weiss E, Uziely B and Grisaru-Granovsky S: PAR1 plays a role in epithelial malignancies: transcriptional regulation and novel signaling pathway. IUBMB Life 63: 397-402, 2011.

14. Tantivejkul K, Loberg RD, Mawocha SC, Day LL, John LS, Pienta BA, Rubin MA and Pienta KJ: PAR1-mediated NFkappaB activation promotes survival of prostate cancer cells through a Bcl-xL-dependent mechanism. J Cell Biochem 96: 641-652, 2005.

15. Shimizu S, Gabazza EC, Hayashi T, Ido M, Adachi Y and Suzuki K: Thrombin stimulates the expression of PDGF in lung epithelial cells. Am J Physiol Lung Cell Mol Physiol 279: L503-L510, 2000.

16. Huang YQ, Li JJ, Hu L, Lee M and Karpatkin S: Thrombin induces increased expression and secretion of VEGF from human FS4 fibroblasts, DU145 prostate cells and CHRF megakaryocytes. Thromb Haemost 86: 1094-1098, 2001.

17. Boire A, Covic L, Agarwal A, Jacques S, Sherifi S, Kuliopulos A: PAR1 is a matrix metalloprotease-1 receptor that promotes invasion and tumorigenesis of breast cancer cells. Cell 120: 303-313, 2005.

18. Poola I, DeWitty RL, Marshalleck JJ, Bhatnagar R, Abraham J and Leffall LD: Identification of MMP-1 as a putative breast cancer predictive marker by global gene expression analysis. Nat Med 11: 481-483, 2005. 
19. Murray GI, Duncan ME, O'Neil P, McKay JA, Melvin WT and Fothergill JE: Matrix metalloproteinase-1 is associated with poor prognosis in oesophageal cancer. J Pathol 185: 256-261, 1998.

20. Migita T, Sato E, Saito K, Mizoi T, Shiiba K, Matsuno S, Nagura $\mathrm{H}$ and Ohtani $\mathrm{H}$ : Differing expression of MMPs-1 and -9 and urokinase receptor between diffuse- and intestinal-type gastric carcinoma. Int J Cancer 84: 74-79, 1999.

21. Yang E, Boire A, Agarwal A, Nguyen N, O'Callaghan K, Tu P Kuliopulos A and Covic L: Blockade of PAR1 signaling with cell-penetrating pepducins inhibits Akt survival pathways in breast cancer cells and suppresses tumor survival and metastasis. Cancer Res 69: 6223-6231, 2009.

22. Gartel AL and Kandel ES: RNA interference in cancer. Biomol Eng 23: 17-34, 2006

23. Kim DH and Rossi JJ: Overview of gene silencing by RNA interference. In: Curr Protoc Nucleic Acid Chem. Beaucage SL (ed) Vol 16. Unit 16.1. Wiley, New York, NY, 2009.

24. Takeshita F and Ochiya T: Therapeutic potential of RNA interference against cancer. Cancer Sci 97: 689-696, 2006.
25. Wu Z, Li X, Zeng Y, Zhuang X, Shen H, Zhu H, Liu H and $\mathrm{Xiao} \mathrm{H}$ : In vitro and in vivo inhibition of MRP gene expression and reversal of multidrug resistance by siRNA. Basic Clin Pharmacol Toxicol 108: 177-184, 2011.

26. Xiao H, Wu Z, Shen H, Luo AL, Yang YF, Li XB and Zhu DY: In vivo reversal of P-glycoprotein-mediated multidrug resistance by efficient delivery of stealth RNAi. Basic Clin Pharmacol Toxicol 103: 342-348, 2008

27. Perrimon N, Ni JQ and Perkins L: In vivo RNAi: today and tomorrow. Cold Spring Harb Perspect Biol 2: a003640, 2010.

28. Seyhan AA: RNAi: a potential new class of therapeutic for human genetic disease. Hum Genet 130: 583-605, 2011.

29. Heller LC, Ugen K and Heller R: Electroporation for targeted gene transfer. Expert Opin Drug Deliv 2: 255-268, 2005.

30. Li S: Electroporation gene therapy: new developments in vivo and in vitro. Curr Gene Ther 4: 309-316, 2004

31. Wells DJ: Gene therapy progress and prospects: electroporation and other physical methods. Gene Ther 11: 1363-1369, 2004. 